\title{
Comparing "F-AV-1451 with CSF t-tau and p-tau for diagnosis of Alzheimer disease
}

Niklas Mattsson, MD, PhD, Ruben Smith, MD, PhD, Olof Strandberg, PhD, Sebastian Palmqvist, MD, PhD, Michael Schöll, PhD, Philip S. Insel, Douglas Hägerström, MD, PhD, Tomas Ohlsson, PhD,

Henrik Zetterberg, MD, PhD, Kaj Blennow, MD, PhD, Jonas Jögi, MD, PhD, and Oskar Hansson, MD, PhD

Neurology ${ }^{\circledR}$ 2018;90:e388-e395. doi:10.1212/WNL.0000000000004887

\section{Abstract}

\section{Objective}

To compare PET imaging of tau pathology with CSF measurements (total tau [t-tau] and phosphorylated tau $[\mathrm{p}$-tau] $)$ in terms of diagnostic performance for Alzheimer disease (AD).

\section{Methods}

We compared t-tau and p-tau and ${ }^{18} \mathrm{~F}-\mathrm{AV}$-1451 in 30 controls, 14 patients with prodromal $\mathrm{AD}$, and 39 patients with Alzheimer dementia, recruited from the Swedish BioFINDER study. All patients with $\mathrm{AD}$ (prodromal and dementia) were screened for amyloid positivity using CSF $\beta$-amyloid 42. Retention of ${ }^{18} \mathrm{~F}$-AV-1451 was measured in a priori specified regions, selected for known associations with tau pathology in $\mathrm{AD}$.

\section{Results}

Retention of ${ }^{18} \mathrm{~F}-\mathrm{AV}$-1451 was markedly elevated in Alzheimer dementia and moderately elevated in prodromal $\mathrm{AD}$. CSF t-tau and p-tau was increased to similar levels in both $\mathrm{AD}$ dementia and prodromal AD. ${ }^{18} \mathrm{~F}-\mathrm{AV}-1451$ had very good diagnostic performance for Alzheimer dementia (area under the receiver operating characteristic curve [AUROC] 1.000), and was significantly better than t-tau (0.876), p-tau (0.890), hippocampal volume (0.824), and temporal cortical thickness (0.860). For prodromal $\mathrm{AD}$, there were no significant AUROC differences between CSF tau and ${ }^{18} \mathrm{~F}-\mathrm{AV}-1451$ measures (0.836-0.939), but MRI measures had lower AUROCs (0.652-0.769).

\section{Conclusions}

$\mathrm{CSF}$ tau and ${ }^{18} \mathrm{~F}-\mathrm{AV}-1451$ have equal performance in early clinical stages of $\mathrm{AD}$, but ${ }^{18} \mathrm{~F}-\mathrm{AV}$ 1451 is superior in the dementia stage, and exhibits close to perfect diagnostic performance for mild to moderate $\mathrm{AD}$.

\section{Classification of evidence}

This study provides Class III evidence that CSF tau and ${ }^{18} \mathrm{~F}-\mathrm{AV}-1451$ PET have similar performance in identifying early $\mathrm{AD}$, and that ${ }^{18} \mathrm{~F}-\mathrm{AV}-1451$ PET is superior to CSF tau in identifying mild to moderate $\mathrm{AD}$.

\author{
Correspondence \\ Dr. Mattsson \\ niklas.mattsson@med.lu.se \\ or Dr. Hansson \\ oskar.hansson@med.lu.se \\ MORE ONLINE \\ $\rightarrow$ Class of Evidence \\ Criteria for rating \\ therapeutic and diagnostic \\ Studies \\ NPub.org/coe
}




\section{Glossary}

$\mathbf{A} \beta=\beta$-amyloid $\mathbf{A D}=$ Alzheimer disease $; \mathbf{A N T}=$ Advanced Normalization Tools; $\mathbf{A U R O C}=$ area under the receiver operating characteristic curve; DSM-III-R = Diagnostic and Statistical Manual of Mental Disorders, 3rd edition, revised; $\mathbf{M C I}=$ mild cognitive impairment; MMSE = Mini-Mental State Examination; $\mathbf{p}$-tau = phosphorylated tau; $\mathbf{Q C}=$ quality control; ROI = region of interest; SUV = standardized uptake value; SUVR = standardized uptake value ratio; $\mathbf{t}$-tau $=$ total tau.

Alzheimer disease $(\mathrm{AD})$ is characterized by the aggregation of $\beta$-amyloid $(A \beta)$ in extracellular plaques and phosphorylated tau (p-tau) in intracellular neurofibrillary aggregates. Tau can be measured in CSF as total tau ( $\mathrm{t}$-tau), which is increased in $\mathrm{AD}$ and in several other neurologic diseases, or as $\mathrm{p}$-tau, which is more specifically increased in $\mathrm{AD} .{ }^{1} \mathrm{PET}$ tracers have made it possible to visualize and quantify tau deposits in vivo. One of these tracers, ${ }^{18} \mathrm{~F}-\mathrm{AV}-1451$ (formerly called ${ }^{18} \mathrm{~F}-\mathrm{T} 8077^{2,3}$ ), binds to tau aggregates in $\mathrm{AD}^{4}$ and differentiates $\mathrm{AD}$ from controls. $^{5-9}$ Preliminary evidence indicates that CSF tau and PET tau measures correlate, ${ }^{6,10}$ but those results stem from populations mainly consisting of controls, including few cases with $\mathrm{AD}$ dementia. A key unresolved question is therefore if CSF and PET tau measures have similar or different diagnostic performance for $\mathrm{AD}$. It is also not clear if CSF and PET tau measures are significantly better than MRI measures of brain structure to identify $\mathrm{AD}$. To address these questions, we compared CSF t-tau and p-tau, ${ }^{18} \mathrm{~F}-\mathrm{AV}$-1451 PET, hippocampal volume, and cortical thickness in $\mathrm{AD}$-associated regions ${ }^{11}$ for diagnosis of $\mathrm{AD}$ at the dementia and prodromal stages of the disease. All patients with prodromal $\mathrm{AD}$ and $\mathrm{AD}$ dementia were screened for amyloid positivity using CSF A $\beta 42$.

\section{Methods}

\section{Participants}

The study population stemmed from 3 cohorts from the prospective and longitudinal Swedish BioFINDER study (biofinder.se). In the present study, we included 30 cognitively normal control participants. They were eligible for inclusion if they (1) were aged $\geq 60$ years old, (2) scored 28-30 points on the Mini-Mental State Examination (MMSE) at the screening visit, (3) did not fulfill the criteria of mild cognitive impairment (MCI) or any dementia, and (4) were fluent in Swedish. The exclusion criteria were (1) presence of significant neurologic or psychiatric disease (e.g., stroke, Parkinson disease, multiple sclerosis, major depression), (2) significant systemic illness making it difficult to participate, (3) refusing lumbar puncture, or (4) substantial alcohol abuse. In the second cohort, 14 patients with $\mathrm{MCI}$ due to $\mathrm{AD}$ (prodromal $\mathrm{AD}$ ) were enrolled at the Memory Clinic of the Skåne University Hospital, Sweden. These participants were eligible for inclusion if they (1) were referred to the memory clinics because of cognitive impairment, (2) did not fulfil the criteria for dementia, (3) scored 24-30 points on the MMSE, (4) had objective memory impairment according to delayed word list recall, (5) were aged 60-80 years, (6) had low CSF A $\beta 42$ levels, ${ }^{12}$ and (7) were fluent in Swedish. The exclusion criteria were (1) cognitive impairment explained by another condition (other than prodromal dementia), (2) a substantial systemic illness making it difficult to participate, (3) refusing lumbar puncture, or (4) substantial alcohol abuse. In the last cohort, we included 39 patients with $\mathrm{AD}$ dementia at baseline, who were recruited at the Memory Clinic, Skåne University Hospital. All patients with dementia met the DSM-III-R criteria for dementia ${ }^{13}$ as well as the National Institute of Neurological and Communicative Disorders and StrokeAlzheimer's Disease and Related Disorders Association criteria for $\mathrm{AD}^{14}$ and had low $\mathrm{CSF} A \beta 42$ levels. The exclusion criteria were (1) substantial systemic illness making it difficult to participate or (2) substantial alcohol abuse. The diagnosis of prodromal $\mathrm{AD}$ and $\mathrm{AD}$ dementia were established by physicians specialized in dementia disorders, who were blinded to the ${ }^{18} \mathrm{~F}-\mathrm{AV}-1451$ PET, CSF $\mathrm{t}$-tau, and CSF $\mathrm{p}$-tau data.

\section{Cognitive measures}

We used the MMSE as a measure of general cognition and the delayed recall memory test from the Alzheimer's Disease Assessment Scale-cognitive subscale (list learning, 10 items) as a measure of memory. ${ }^{15}$

\section{CSF biomarkers}

CSF samples were derived from lumbar puncture. Samples were analyzed at the Clinical Neurochemistry Laboratory in Mölndal, Sweden, for t-tau, p-tau, and A $\beta 42$ using commercially available ELISAs (INNOTEST; Fujiribio, Ghent, Belgium). All CSF samples were analyzed using clinical practice procedures, with analyses performed by boardcertified technicians blinded to clinical data, following detailed procedures to assure analytical precision and long-term stability of the biomarkers, including batch bridging between old and new batches of ELISA plates, general laboratory procedures (e.g., calibration of pipettes and preventive service of instruments), and strict criteria for approval of calibration curves and internal quality control (QC) samples, following the Westgard multi rules, as described previously in detail. ${ }^{16}$ The approval limits for the 2 internal QC CSF samples run at 2 positions on each plate was $12.0 \%$ for $A \beta 42,9.3 \%$ for $t$-tau, and $9.8 \%$ for $\mathrm{p}$-tau for the normal QC sample, and $11.0 \%$ for $\mathrm{A} \beta 42,10.0 \%$ for $\mathrm{t}$-tau, and $9.8 \%$ for $\mathrm{p}$-tau for the $\mathrm{AD}$-like $\mathrm{QC}$ sample. For CSF $A \beta 42$, we used a cutoff of $<650 \mathrm{ng} / \mathrm{L}$ to identify $A \beta$-positive participants, based on our previous comparisons between CSF A $\beta 42$ and A $\beta$ PET imaging. ${ }^{16}$ All patients with prodromal $\mathrm{AD}$ and patients with $\mathrm{AD}$ dementia were screened for $A \beta$ positivity before ${ }^{18} \mathrm{~F}-\mathrm{AV}-1451$ PET 
scanning. The control population was enriched for $A \beta$ pathology, by inclusion of $15 \mathrm{~A} \beta$-positive and $15 \mathrm{~A} \beta$-negative participants before ${ }^{18} \mathrm{~F}-\mathrm{AV}-1451$ PET scanning.

\section{MRI and processing}

T1-weighted imaging was performed on a $3 \mathrm{~T}$ magnetic resonance scanner (Siemens Tim Trio 3T; Siemens Medical Solutions, Erlangen, Germany), producing a highresolution anatomic magnetization-prepared rapid gradient echo image (repetition time 1,950 ms, echo time $3.4 \mathrm{~ms}$, $1 \mathrm{~mm}$ isotropic voxels, and 178 slices) for further use in volumetric analysis, template normalization, and coregistrations. The anatomic scan was normalized to Montreal Neurological Institute 152 space $^{17}$ with a diffeomorphic transform and the Advanced Normalization Tools (ANT) toolbox ${ }^{18}$ for further use in the PET processing pipeline (see below; ANT was used for all coregistrations). Cortical reconstruction and volumetric segmentation were performed with the Freesurfer image analysis pipeline v5.3 (surfer.nmr. mgh.harvard.edu/). We used the average cortical thickness in temporal lobe regions (including the FreeSurfer regions of interest [ROIs] entorhinal, fusiform, inferior temporal, and middle temporal cortex, based on reference 11), and hippocampal volume (averaged between right and left hemisphere).

\section{Tau PET imaging and processing}

${ }^{18}$ F-AV-1451 was synthesized at Skåne University Hospital, Lund, as described previously. ${ }^{19}$ PET scans were performed on a GE Discovery 690 PET scanner (General Electric Medical Systems, Bensalem, PA) as dynamic scans using LIST-mode 80-120 minutes after a bolus injection of 370 $\mathrm{MBq}$ of ${ }^{18} \mathrm{~F}-\mathrm{AV}-1451$. Low-dose CT scans for attenuation correction were performed in the same patient position immediately prior to the PET scans. PET data were reconstructed into 5-minute frames using an iterative Vue Point HD algorithm with 6 subsets, 18 iterations with 3-mm filter, and no time-of-flight correction. The dynamic scans were motion corrected using AFNI's 3 dvolreg, ${ }^{20}$ time-averaged and rigidly coregistered to the skull-stripped MRI scan. Partial volume error correction was performed using the geometric transfer method as described in reference 21 using the FreeSurfer parcellations, smoothed with 5-mm full width at half maximum to calculate transfers across ROI borders. The FreeSurfer parcellation in the magnetic resonance space of the anatomic scan was then applied to the processed, coregistered, and time-averaged PET image to extract regional uptake values. We created ${ }^{18} \mathrm{~F}-\mathrm{AV}-1451$ standardized uptake value (SUV) images based on mean uptake over $80-120$ minutes postinjection normalized to uptake in a gray matter-masked cerebellum reference region to create voxelwise SUV ratio (SUVR) images in each participant's MRI native space. ${ }^{19}$

\section{Regional PET analyses}

We performed ${ }^{18} \mathrm{~F}-\mathrm{AV}-1451$ PET analyses with a priori defined ROI, as proposed by Cho et al. ${ }^{22}$ and Jack et al. ${ }^{23}$ Cho et al. $^{22}$ described a protocol to aggregate FreeSurfer ROIs in different tau stages, which was overall similar to the staging system suggested by Braak and Braak. ${ }^{24}$ We used this protocol to define a set of nonoverlapping ROIs corresponding to tau stages I-II, III, IV, V, and VI (table 1). To obtain an overall ${ }^{18} \mathrm{~F}-\mathrm{AV}-1451$ PET tau measure, we merged the signal from tau stages I-V. As an alternative overall tau measure, we merged the signal from tau stages regions I-IV, which corresponds to an aggregation protocol suggested by Jack et al. ${ }^{23}$ For merged regions, the signal was calculated as the sum of the volumeadjusted regional ${ }^{18} \mathrm{~F}-\mathrm{AV}-1451$ PET signals.

\section{Statistical analyses}

Biomarker levels were compared between diagnostic groups by linear regression, adjusted for age. Sex was also explored as a covariate, but was left out since it was nonsignificant and did not affect the results.

Diagnostic performance of biomarkers was quantified by area under the ROC (AUROC) analysis. AUROCs were compared between biomarkers using a bootstrap method with 2,000 iterations. For all tau biomarkers, we determined cutoffs by the Youden index (J, which maximizes the combination of sensitivity and specificity). We calculated sensitivities, specificities, and overall accuracies (proportion of correctly classified participants) at these cutoffs.

All statistical tests were 2-sided. Significance was determined at $p<0.05$. All statistics were done using $\mathrm{R}$ (v. 3.2.3, The $\mathrm{R}$ Foundation for Statistical Computing). The pROC package (v.1.8) was used for AUROC analyses.

\section{Primary research questions}

Do the diagnostic performances differ between CSF tau measures and ${ }^{18} \mathrm{~F}-\mathrm{AV}-1451$ PET for early $\mathrm{AD}$ (prodromal disease stage) and for $\mathrm{AD}$ dementia (mild to moderate disease stage)? This study provides Class III evidence that CSF tau measures and ${ }^{18} \mathrm{~F}-\mathrm{AV}-1451$ PET have similar performance for identifying early (prodromal) $\mathrm{AD}$, and that ${ }^{18} \mathrm{~F}-\mathrm{AV}-1451 \mathrm{PET}$

Table 1 Regions used for different tau stages

\begin{tabular}{ll}
\hline $\begin{array}{l}\text { Tau } \\
\text { stage }\end{array}$ & FreeSurfer regions of interest \\
\hline I-II & Entorhinal \\
\hline III & Parahippocampal, fusiform, amygdala \\
\hline IV & Inferior temporal, middle temporal \\
\hline V & $\begin{array}{l}\text { Posterior cingulate, caudal anterior cingulate, rostral anterior } \\
\text { cingulate, precuneus, inferior parietal, superior parietal, insula, } \\
\text { supramarginal, lingual, superior temporal, medial } \\
\text { orbitofrontal, rostral middle frontal, lateral orbitofrontal, } \\
\text { caudal middle frontal, superior frontal, lateral occipital }\end{array}$ \\
\hline VI & \begin{tabular}{l} 
Precentral gyrus, postcentral gyrus, paracentral gyrus \\
\hline
\end{tabular}
\end{tabular}

Tau stage regions based on references 22 and 23 . 
is superior to CSF tau measures in identifying mild to moderate $\mathrm{AD}$.

\section{Standard protocol approvals, registrations, and patient consents}

All participants gave written informed consent to participate in the study. Ethical approval was given by the Ethical Committee of Lund University, Sweden, and all the methods were carried out in accordance with the approved guidelines. ${ }^{18}$ F-AV-1451 PET imaging approval was obtained from the Swedish Medicines and Products Agency and the local Radiation Safety Committee at Skåne University Hospital, Sweden.

\section{Results}

\section{${ }^{18} \mathrm{~F}-\mathrm{AV}-1451, \mathrm{CSF}$ tau, and MRI biomarkers by diagnosis}

Demographics are presented in table 2. Compared to controls, the ${ }^{18} \mathrm{~F}-\mathrm{AV}-1451$ retention was elevated in $\mathrm{AD}$ dementia in all tau stages, and in prodromal $\mathrm{AD}$ in tau stage $\mathrm{I}-\mathrm{V}$ regions (figure 1). The ${ }^{18} \mathrm{~F}-\mathrm{AV}$-1451 retention was also elevated in $\mathrm{AD}$ dementia compared to prodromal $\mathrm{AD}$ in all tau stages except stage IV. The differences between the diagnostic groups in ${ }^{18} \mathrm{~F}$-AV-1451 were similar for the merged stage I-IV and $\mathrm{I}-\mathrm{V}$ regions (figure $2, \mathrm{~A}$ and $\mathrm{B}$ ). Patients with $\mathrm{AD}$ dementia and prodromal $\mathrm{AD}$ had higher CSF t-tau and p-tau than controls, but there were no differences between patients with $\mathrm{AD}$ dementia and patients with prodromal $\mathrm{AD}$ in CSF tau measures (figure 2, $\mathrm{C}$ and $\mathrm{D}$ ). Patients with $\mathrm{AD}$ dementia and prodromal $\mathrm{AD}$ had smaller hippocampi and thinner cortical thickness of the temporal lobe than controls, and

Table 2 Study demographics

\begin{tabular}{llll}
\hline & Controls & $\begin{array}{l}\text { Prodromal } \\
\text { AD }\end{array}$ & $\begin{array}{l}\text { AD } \\
\text { dementia }\end{array}$ \\
\hline $\mathbf{N}$ & 30 & 14 & 39 \\
\hline Age, $\mathbf{y}$ & $74.7(5.5)$ & $71.6(6.3)$ & $71.3(7.2)$ \\
\hline Sex, F/M & $15 / 15$ & $10 / 4$ & $18 / 21$ \\
\hline Education, $\mathbf{y}$ & $11.3(3.9)$ & $11.5(3.8)$ & $11.9(3.4)$ \\
\hline MMSE & $29.3(0.8)$ & $24.9(2.6)$ & $21.1(5.0)$ \\
\hline ADAS-Cog delayed recall & $2.2(1.4)$ & $6.3(2.4)$ & $8.4(2.0)$ \\
\hline CSF A $\beta 42$, ng/L & $682(188)$ & $432(83)$ & $393(115)$ \\
\hline CSF A $\beta 42, \pm$ & $15 / 15$ & $14 / 0(100 \%)$ & $39 /$ \\
\hline $\begin{array}{l}\text { Time between LP and tau } \\
\text { PET, mo }\end{array}$ & $6.5(8.9)$ & $18.6(16.0)$ & $18.7(18.2)$ \\
\hline
\end{tabular}

Abbreviations: $A \beta=\beta$-amyloid; $A D=$ Alzheimer disease; $A D A S-C o g=$ Alzheimer's Disease Assessment Scale-cognitive subscale; LP = lumbar puncture; MMSE = Mini-Mental State Examination.

Continuous data shown as mean (SD). patients with $\mathrm{AD}$ dementia had smaller hippocampi than patients with prodromal $\mathrm{AD}$ (figure 2, $\mathrm{E}$ and $\mathrm{F}$ ).

\section{Diagnostic performances of tau biomarkers}

We calculated AUROCs for ${ }^{18} \mathrm{~F}-\mathrm{AV}-1451$ in tau stage I-IV and stage I-V, CSF t-tau and p-tau, hippocampal volume, and temporal cortical thickness (figure 3). ${ }^{18} \mathrm{~F}-\mathrm{AV}-1451$ had almost perfect separation for $\mathrm{AD}$ dementia vs controls. The AUROCs were significantly higher for ${ }^{18} \mathrm{~F}-\mathrm{AV}$-1451 measures than for CSF t-tau, p-tau, and MRI measures, but there were no significant differences in AUROCs between CSF T-tau, p-tau, and MRI measures. For patients with prodromal $A D$ vs controls, there were no significant differences in AUROCs between the tau biomarkers, but ${ }^{18} \mathrm{~F}-\mathrm{AV}-1451$ in tau stage I-IV, CSF t-tau, and CSF p-tau all had significantly higher AUROCs than hippocampal volume. The PET and CSF tau biomarkers also tended to have higher AUROCs than temporal lobe cortical thickness for prodromal $\mathrm{AD}$, but the differences were not significant. The AUROC results were similar when not performing partial volume correction of the ${ }^{18} \mathrm{~F}-\mathrm{AV}$-1451 images, and when adjusting for age and time lag between PET and lumbar puncture (data not shown). We also compared biomarker AUROCS for preclinical $A D$ $(\mathrm{A} \beta$-positive controls, $\mathrm{n}=15$ ) vs prodromal $\mathrm{AD}$ and $\mathrm{AD}$ dementia. The findings were similar to when including all controls (supplementary analysis [links.lww.com/WNL/A93] and figure e-1, [links.lww.com/WNL/A91]).

We next determined optimal cutoffs for the PET and CSF tau biomarkers using the Youden index. For patients with $\mathrm{AD}$ dementia vs controls, these cutoffs were CSF $\mathrm{t}$-tau $>624 \mathrm{ng} / \mathrm{L}$ (sensitivity 69\%, specificity 90\%), CSF p-tau $>72 \mathrm{ng} / \mathrm{L}$ (sensitivity $72 \%$, specificity $93 \%$ ), ${ }^{18} \mathrm{~F}-\mathrm{AV}$-1451 tau stage I-IV $>1.54$ SUVR (sensitivity 97\%, specificity 100\%), and

${ }^{18}$ F-AV-1451 tau stage I-V >1.37 SUVR (sensitivity 92\%, specificity $100 \%$ ). For prodromal $\mathrm{AD}$ vs controls, the cutoffs were CSF t-tau $>504 \mathrm{ng} / \mathrm{L}$ (sensitivity $86 \%$, specificity $70 \%$ ), CSF p-tau $>73 \mathrm{ng} / \mathrm{L}$ (sensitivity 79\%, specificity 93\%), ${ }^{18} \mathrm{~F}-\mathrm{AV}-1451$ tau stage I-IV $>1.41$ SUVR (sensitivity 79\%, specificity $87 \%$ ), and ${ }^{18} \mathrm{~F}-\mathrm{AV}-1451$ tau stage I-V $>1.43$ SUVR (sensitivity 57\%, specificity 100\%). See table e-1 (links.lww. $\mathrm{com} / \mathrm{WNL} / \mathrm{A} 92$ ) for a summary of these data.

\section{Discussion}

We found that ${ }^{18} \mathrm{~F}-\mathrm{AV}-1451$ tau PET imaging was superior to CSF tau biomarkers for diagnosis of mild to moderate $\mathrm{AD}$ dementia vs controls, with almost perfect separation between groups. In prodromal $\mathrm{AD}$, when some patients still lacked widespread tau pathology, ${ }^{18} \mathrm{~F}-\mathrm{AV}-1451$ PET and CSF tau biomarkers had comparable diagnostic performance.

Studies comparing CSF tau biomarkers with PET tau imaging for diagnosis of $\mathrm{AD}$ are rare. Our findings suggest that the relationship between CSF and PET tau biomarkers for diagnosis differs by disease stage in $\mathrm{AD}$. This supports a model where CSF tau biomarkers are primarily useful as disease state 


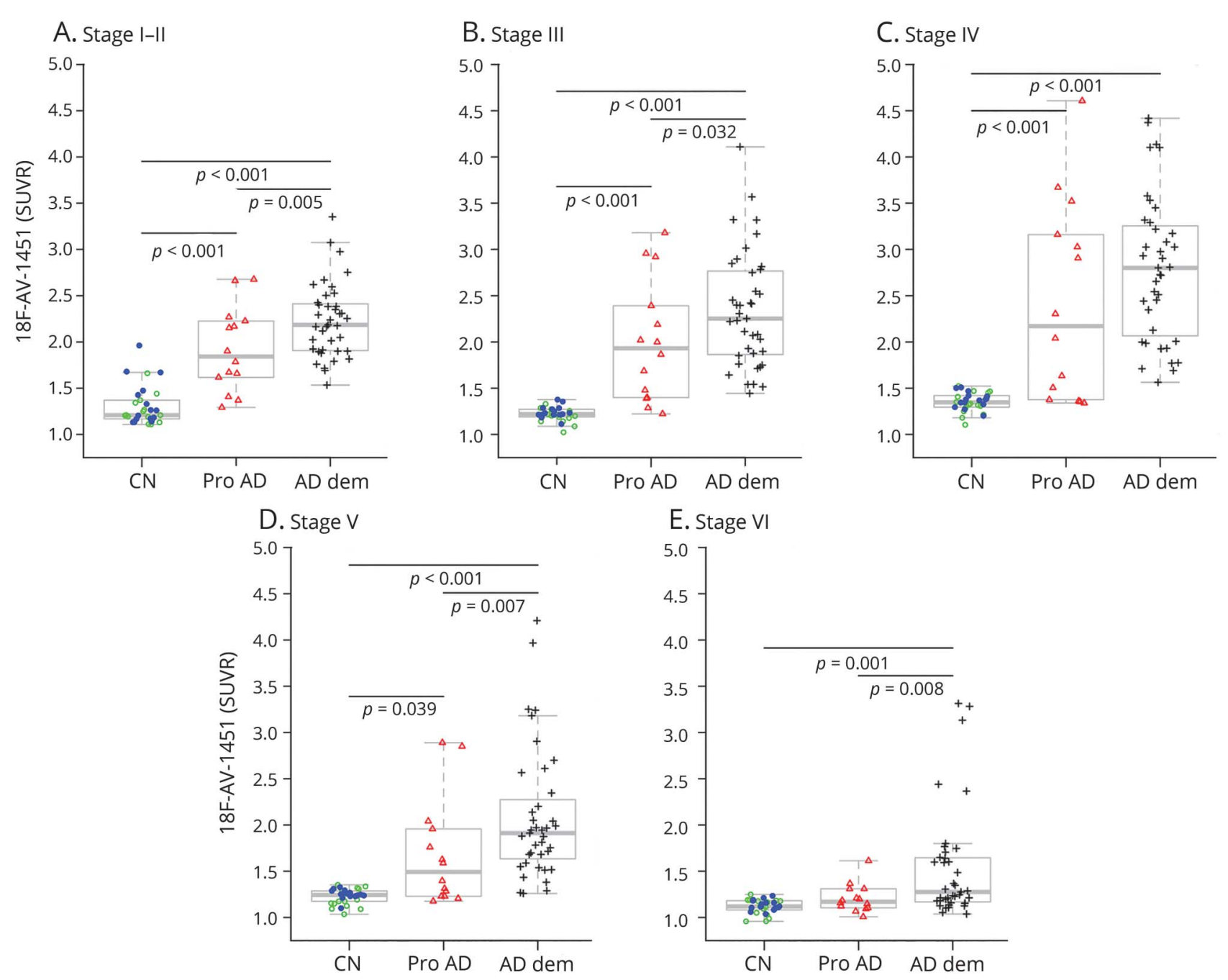

(A-E) ${ }_{18} \mathrm{~F}-\mathrm{AV}$-1451 signal in different tau stage regions. Diagnostic groups (controls [CN], prodromal Alzheimer disease [Pro AD], and Alzheimer disease dementia [AD dem]) were compared by linear regression, adjusted for age. The controls are coded by amyloid status (amyloid-negative, green open circles; amyloid-positive, blue dots). SUVR = standardized uptake value ratio.

biomarker, i.e., they indicate presence or absence of $\mathrm{AD}$, but they may be less useful as stage biomarkers during the transition from prodromal $\mathrm{AD}$ to dementia. In contrast, ${ }^{18} \mathrm{~F}-\mathrm{AV}$ 1451 imaging may be useful both as a state and a stage biomarker, since increased ${ }^{18} \mathrm{~F}-\mathrm{AV}$ - 1451 is associated with $\mathrm{AD}$ already at the prodromal stage, and provides increased separation towards controls in the dementia stage of the disease. We also included MRI measures of brain structure (hippocampal volume and temporal lobe cortical thickness), which had lower AUROC than ${ }^{18} \mathrm{~F}-\mathrm{AV}$-1451 for AD dementia. For prodromal $\mathrm{AD}$, hippocampal volume had significantly lower AUROC than PET and CSF tau measures, and there was also a tendency for lower AUROC for temporal lobe cortical thickness compared to the tau measures.

At the dementia stage, ${ }^{18} \mathrm{~F}-\mathrm{AV}-1451$ was superior to CSF tau biomarkers for $\mathrm{AD}$ diagnosis. The diagnostic performance of CSF tau biomarkers may be confounded both by the physiologic between-person variability in CSF tau concentrations and by release of tau due to nonspecific neuronal injury. ${ }^{25}$ Another possibility that needs to be tested by longitudinal studies is that CSF tau may be more sensitive than ${ }^{18} \mathrm{~F}-\mathrm{AV}-1451$ to very early pathologic tau-related changes. For example, release of neuronal tau may be involved in interneuronal transmission of tau pathology, ${ }^{26}$ which hypothetically may occur before tau pathology is detected by ${ }^{18} \mathrm{~F}$ AV-1451 imaging. Similarly, we have previously shown that CSF biomarkers may be more sensitive to $A \beta$ pathology compared to PET imaging. ${ }^{27}$ The fact that CSF tau measures did not differ between prodromal $\mathrm{AD}$ and $\mathrm{AD}$ dementia suggests that these biomarkers plateau at the prodromal stage of the disease. In contrast, the ${ }^{18} \mathrm{~F}-\mathrm{AV}-1451$ signal was higher in the $\mathrm{AD}$ dementia than in the prodromal $\mathrm{AD}$ group, which likely reflects a continuous accumulation of tau as the disease progresses. One important difference between CSF and PET tau measurements is that ${ }^{18} \mathrm{~F}-\mathrm{AV}-1451$ makes it possible to 

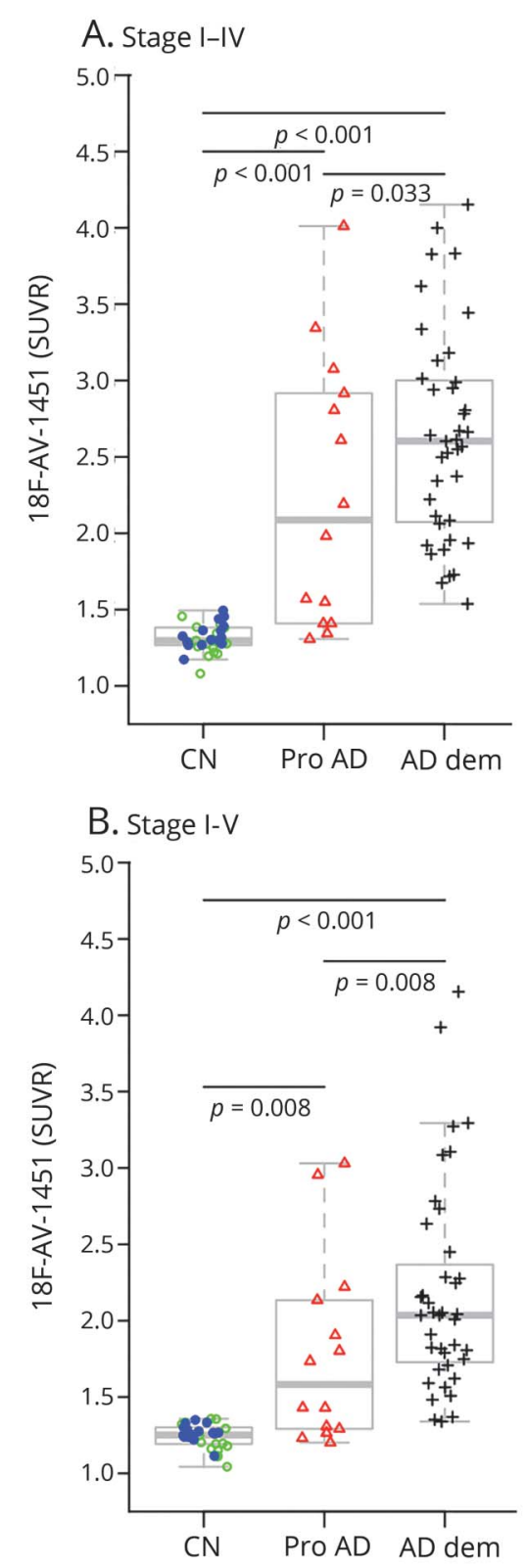

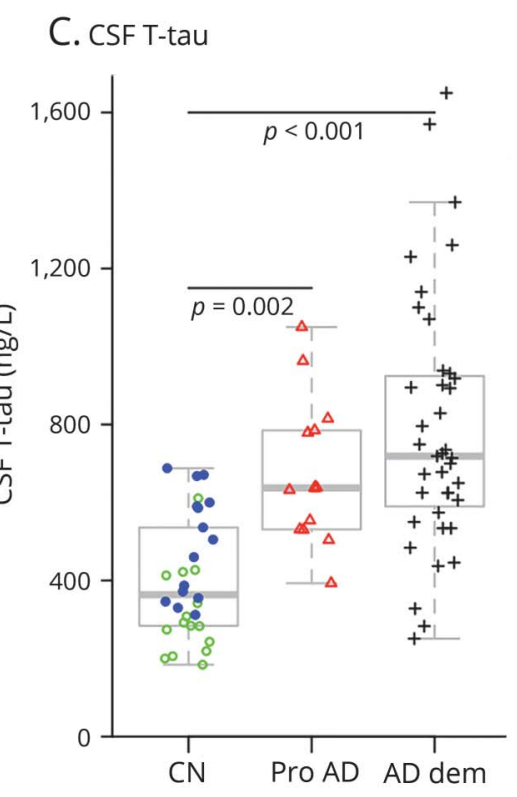

D. CSF P-tau

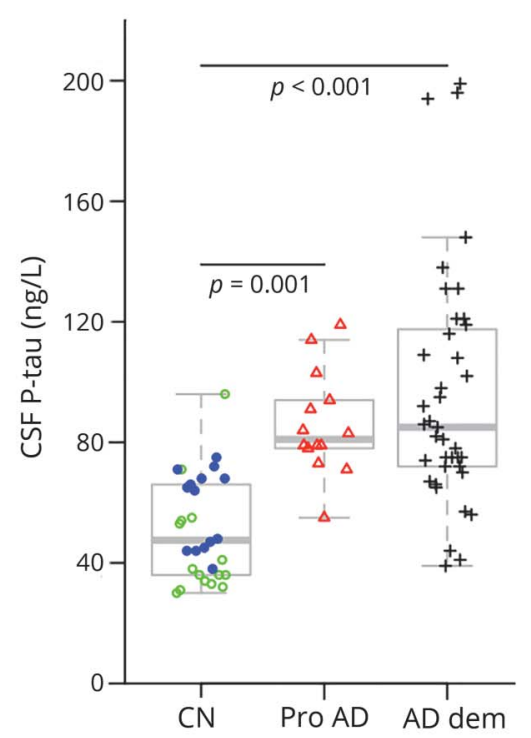

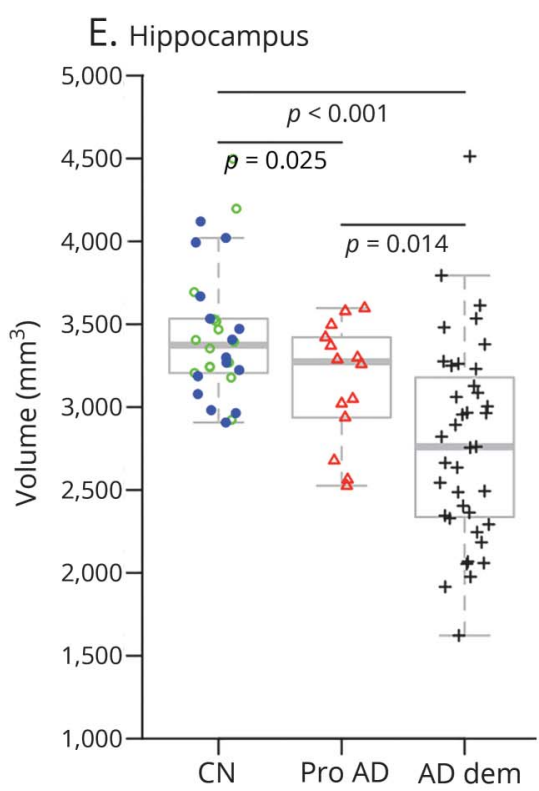

F. Temporal cortical

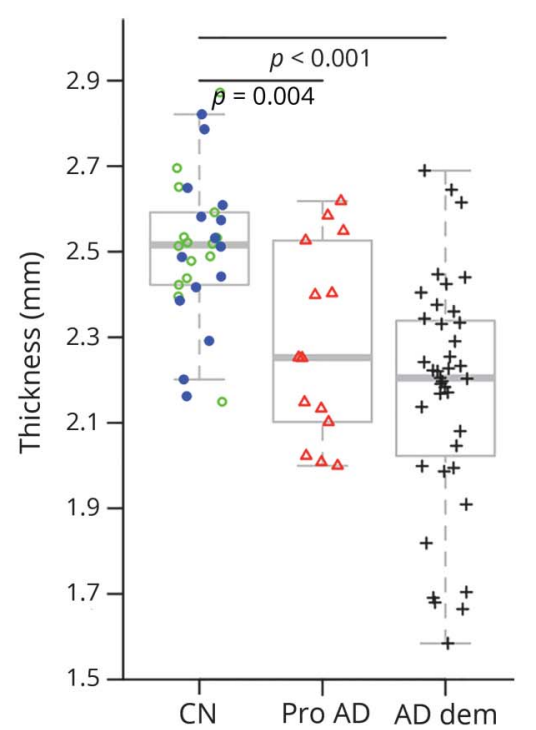

(A, B) ${ }_{18}$ F-AV-1451 signal in tau stage regions I-IV and tau stage I-V. (C, D) CSF total tau (t-tau) and phosphorylated tau (p-tau). (E, F) Hippocampal volume and cortical thickness in temporal lobe regions. Diagnostic groups (controls [CN], prodromal Alzheimer disease [Pro AD], and Alzheimer disease dementia [AD dem]) were compared by linear regression, adjusted for age. The controls are coded by amyloid status (amyloid-negative, green open circles; amyloidpositive, blue dots).

track a potential spread of tau to new brain regions. Some regions may be affected later in the disease process (e.g., tau stage VI regions may be affected after tau stage $\mathrm{V}$ regions). This may explain why the latest stages show less separation between diagnostic groups than the earlier stages.

We did not find different results for CSF t-tau and p-tau, despite the fact that CSF p-tau has been suggested to be more closely related to brain tau pathology than CSF t-tau. ${ }^{1}$ However, we note that histopathology studies have found correlations for both CSF $t$-tau and $p$-tau with tangle load, ${ }^{28-30}$ which is in agreement with our finding that both
CSF t-tau and p-tau had similar diagnostic performance as ${ }^{18} \mathrm{~F}-\mathrm{AV}-1451$.

One limitation is the lack of neuropathologic confirmation of tau pathology. Previous studies have found strong correlations between ${ }^{18} \mathrm{~F}-\mathrm{AV}$-1451 PET and tau aggregates consisting of combined $4 \mathrm{R}$ and $3 \mathrm{R}$ tau, ${ }^{31}$ and some studies have found correlations between CSF tau and brain tau pathology ${ }^{28-30}$ (but not all studies have confirmed this ${ }^{32}$ ). Another limitation is that we only included patients with prodromal $\mathrm{AD}$ and patients with $\mathrm{AD}$ dementia with biomarker evidence of amyloid pathology. This was done because modern research 


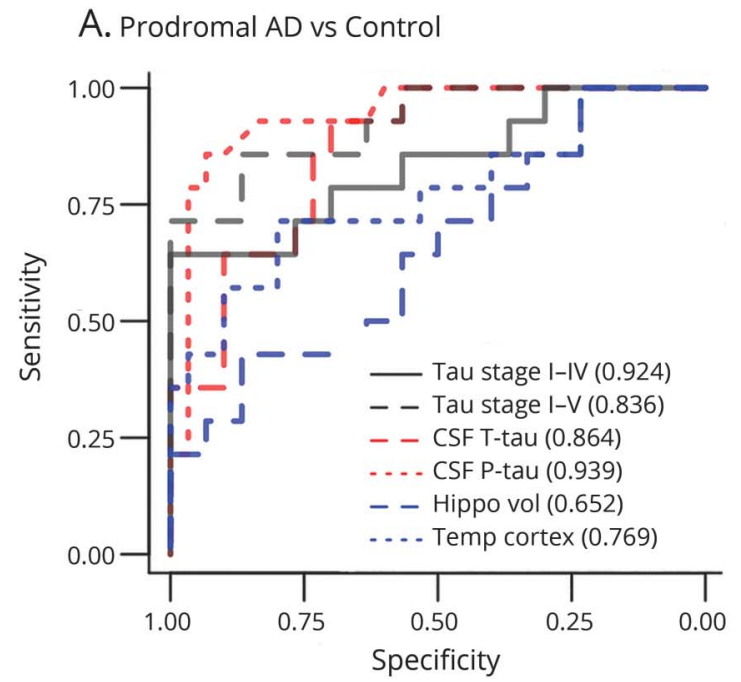

B. AD dementia vs Control

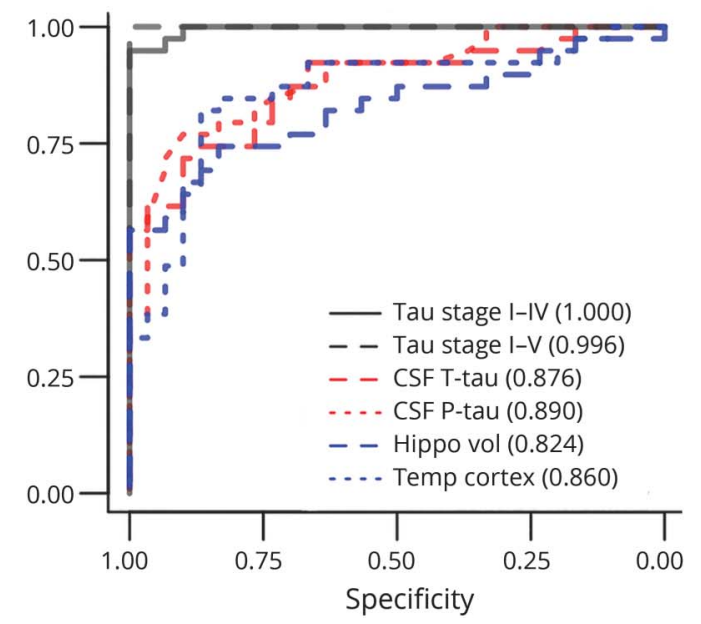

AUROC analyses for the ${ }_{18} \mathrm{~F}-\mathrm{AV}$-1451 signal from the tau stage region I-IV, tau stage region I-V, CSF total tau (t-tau) and phosphorylated tau (p-tau), hippocampal volume, and temporal lobe cortical thickness, to differentiate prodromal Alzheimer disease (AD) (A) and AD dementia (B) from controls. AUROCs are shown in the legends. AUROCs for hippocampal volume were adjusted for intracranial volume. For prodromal AD vs controls, tau stage I-IV, CSF t-tau, and CSF p-tau had higher AUROCs than hippocampal volume ( $p=0.0055 ; p=0.024 ; p=0.0065)$. For AD dementia vs controls, tau stage I-V and tau stage I-IV PET had higher AUROCs than CSF t-tau $(p=0.0033 ; p=0.0025)$, CSF $p$-tau $(p=0.0040 ; p=0.0038)$, hippocampal volume $(p<0.001 ; p<0.001)$, and temporal lobe cortical thickness $(p=0.0034 ; p=0.0038)$.

criteria emphasize amyloid biomarkers for a diagnosis of $\mathrm{AD},{ }^{33,34}$ but we acknowledge that it restricts the generalizability to patients with $\mathrm{AD}$ with evidence of amyloid pathology. Future studies may include a more diverse patient population, including patients with other dementias. We also acknowledge that both CSF tau and ${ }^{18} \mathrm{~F}-\mathrm{AV}-1451$ may be susceptible to measurement errors. The CSF tau measurements were done according to the Alzheimer's Association's guidelines, and the coefficients of variation were $<10 \%$. The ${ }^{18} \mathrm{~F}$-AV-1451 signal may be susceptible to off-target binding in several locations. One prominent off-target site is the choroid plexus, which is very close to the hippocampal formation. Partly because of this, we did not include the ${ }^{18} \mathrm{~F}-\mathrm{AV}-1451$ signal in the hippocampus in this study.

We used the cerebellar gray matter as reference region for ${ }^{18} \mathrm{~F}-\mathrm{AV}-1451$, but the choice of an optimal reference region is not straightforward. There may be minor off-target binding of ${ }^{18} \mathrm{~F}-\mathrm{AV}-1451$ in the rostral part of cerebellum, which may decrease the ability of ${ }^{18} \mathrm{~F}-\mathrm{AV}-1451$ to separate patients with $\mathrm{AD}$ from controls. However, despite this potential source of variability, we demonstrated almost $100 \%$ separation between $\mathrm{AD}$ dementia and controls. In our view, this provides proof-ofprinciple of the superior diagnostic performance of ${ }^{18} \mathrm{~F}-\mathrm{AV}$ 1451 compared to CSF tau measures. Other options for reference regions are problematic. Cerebellar white matter may have a relatively strong nonspecific binding. Cerebral white matter could be used, but the included ROIs then need to be clearly separated from the off-target binding regions in the basal ganglia/thalamus. Future studies may evaluate whether other reference regions would improve the results further.
We found that ${ }^{18} \mathrm{~F}$-AV-1451 PET imaging has superior diagnostic performance compared to CSF tau for $\mathrm{AD}$ in the dementia stage, but the 2 tau biomarker modalities have equal performance for prodromal $\mathrm{AD}$. Future studies may compare longitudinal CSF and PET tau measures to clarify how these measures may develop over time, and how they may respond to disease-modifying treatment in $\mathrm{AD}$.

\section{Author contributions}

Drafting the manuscript: N.M. Revising the manuscript for content: all authors. Study concept or design: N.M., O.H. Analysis or interpretation of data: N.M., O.H. Contribution of vital reagents/tools/patents: K.B., H.Z. Acquisition of data: R.S., O.S., D.H., T.O., J.J., H.Z., K.B. Statistical analysis: N.M. and P.I. Study supervision or coordination: N.M., O.H. Obtaining funding: N.M., O.H.

\section{Study funding}

Work in the authors' laboratory was supported by the European Research Council, the Swedish Research Council, the Strategic Research Area MultiPark (Multidisciplinary Research in Parkinson's disease) at Lund University, the Swedish Brain Foundation, the Skåne University Hospital Foundation, the Swedish Alzheimer Foundation, the Marianne and Marcus Wallenberg Foundation, the Swedish federal government under the ALF agreement, the Greta and Johan Kock Foundation for Medical Research, the Thelma Zoega foundation for medical research, the Bundy Academy, the Torsten Söderberg Foundation, and the Magnus Bergwall Foundation. The funding sources had no role in the design and conduct of the study, in the collection, analysis, or 
interpretation of the data, or in the preparation, review, or approval of the manuscript. The precursor of AV-1451 was provided by Avid Radiopharmaceuticals.

\section{Disclosure}

N. Mattsson, R. Smith, O. Strandberg, S. Palmqvist, M. Schöll, P.S. Insel, D. Hägerström, and T. Ohlsson report no disclosures relevant to the manuscript. H. Zetterberg has served on advisory boards for Roche, Pharmasum, and Eli Lilly. Dr. Zetterberg is a cofounder of Brain Biomarker Solutions in Gothenburg AB, a GU Venture-based platform company at the University of Gothenburg. K. Blennow has served on advisory boards or as a consultant for Alzheon, Eli Lilly, Fujirebio Europe, IBL International, Novartis, and Roche Diagnostics. Dr. Blennow is a cofounder of Brain Biomarker Solutions in Gothenburg AB, a GU Venture-based platform company at the University of Gothenburg. J. Jögi reports no disclosures relevant to the manuscript. O. Hansson has served on advisory boards for Eli Lilly and received research support from GE Healthcare and Hoffmann La-Roche. Go to Neurology.org/N for full disclosures.

Received January 24, 2017. Accepted in final form October 5, 2017.

\section{References}

1. Blennow K, Hampel H, Weiner M, Zetterberg H. Cerebrospinal fluid and plasma biomarkers in Alzheimer disease. Nat Rev Neurol 2010;6:131-144.

2. Chien DT, Bahri S, Szardenings AK, et al. Early clinical PET imaging results with the novel PHF-tau radioligand [F-18]-T807. J Alzheimers Dis 2013;34:457-468.

3. Xia C-F, Arteaga J, Chen G, et al. [18F]T807, a novel tau positron emission tomography imaging agent for Alzheimer's disease. Alzheimers Dement 2013;9: 666-676.

4. Marquié $\mathrm{M}$, Normandin $\mathrm{MD}$, Vanderburg $\mathrm{CR}$, et al. Validating novel tau positron emission tomography tracer [F-18]-AV-1451 (T807) on postmortem brain tissue. Ann Neurol 2015;78:787-800.

5. Brier MR, Gordon $B$, Friedrichsen $K$, et al. Tau and $A \beta$ imaging, CSF measures, and cognition in Alzheimer's disease. Sci Transl Med 2016;8:338ra66.

6. Gordon BA, Friedrichsen K, Brier M, et al. The relationship between cerebrospinal fluid markers of Alzheimer pathology and positron emission tomography tau imaging. Brain J Neurol 2016;139:2249-2260.

7. Johnson KA, Schultz A, Betensky RA, et al. Tau positron emission tomographic imaging in aging and early Alzheimer disease. Ann Neurol 2016;79:110-119.

8. Schöll M, Lockhart SN, Schonhaut DR, et al. PET imaging of tau deposition in the aging human brain. Neuron 2016;89:971-982.

9. Schwarz AJ, Yu P, Miller BB, et al. Regional profiles of the candidate tau PET ligand $18 \mathrm{~F}-\mathrm{AV}-1451$ recapitulate key features of Braak histopathological stages. Brain J Neurol 2016;139:1539-1550.

10. Chhatwal JP, Schultz AP, Marshall GA, et al. Temporal T807 binding correlates with CSF tau and phospho-tau in normal elderly. Neurology 2016;87:920-926.
11. Jack CR, Wiste HJ, Weigand SD, et al. Different definitions of neurodegeneration produce similar amyloid/neurodegeneration biomarker group findings. Brain 2015; 138:3747-3759.

12. Palmqvist S, Zetterberg H, Mattsson N, et al. Detailed comparison of amyloid PET and CSF biomarkers for identifying early Alzheimer disease. Neurology 2015;85:1240-1249.

13. American Psychiatric Association: Work Group to Revise DSM-III. Diagnostic and Statistical Manual of Mental Disorders: DSM-III-R, 3rd ed. Washington, DC: American Psychiatric Association; 1987.

14. McKhann G, Drachman D, Folstein M, Katzman R, Price D, Stadlan EM. Clinical diagnosis of Alzheimer's disease: report of the NINCDS-ADRDA work group under the auspices of Department of Health and Human Services Task Force on Alzheimer's disease. Neurology 1984;34:939-944.

15. Rosen WG, Mohs RC, Davis KL. A new rating scale for Alzheimer's disease. Am J Psychiatry 1984;141:1356-1364.

16. Palmqvist $\mathrm{S}$, Zetterberg $\mathrm{H}$, Blennow $\mathrm{K}$, et al. Accuracy of brain amyloid detection in clinical practice using cerebrospinal fluid $\beta$-amyloid 42: a cross-validation study against amyloid positron emission tomography. JAMA Neurol 2014;71:1282-1289.

17. Grabner G, Janke AL, Budge MM, Smith D, Pruessner J, Collins DL. Symmetric atlasing and model based segmentation: an application to the hippocampus in older adults. Med Image Comput Comput Assist Interv 2006;9:58-66.

18. Avants BB, Tustison NJ, Stauffer M, Song G, Wu B, Gee JC. The Insight ToolKit image registration framework. Front Neuroinform 2014;8:44.

19. Hahn A, Schain M, Erlandsson M, et al. Modeling strategies for quantification of in vivo ${ }^{18} \mathrm{~F}$-AV1451 binding in patients with tau pathology. J Nucl Med 2017;58:623-631.

20. Cox RW. AFNI: software for analysis and visualization of functional magnetic resonance neuroimages. Comput Biomed Res 1996;29:162-173.

21. Rousset OG, Ma Y, Evans AC. Correction for partial volume effects in PET: principle and validation. J Nucl Med 1998;39:904-911.

22. Cho H, Choi JY, Hwang MS, et al. In vivo cortical spreading pattern of tau and amyloid in the Alzheimer disease spectrum. Ann Neurol 2016;80:247-258.

23. Jack CR Jr, Wiste HJ, Weigand SD, et al. Defining imaging biomarker cut points for brain aging and Alzheimer's disease. Alzheimers Dement 2017;13:205-216.

24. Braak H, Braak E. Neuropathological stageing of Alzheimer-related changes. Acta Neuropathol 1991;82:239-259.

25. Mattsson N. CSF biomarkers in neurodegenerative diseases. Clin Chem Lab Med 2011;49:345-352.

26. Maia LF, Kaeser SA, Reichwald J, et al. Changes in amyloid- $\beta$ and tau in the cerebrospinal fluid of transgenic mice overexpressing amyloid precursor protein. Sci Transl Med 2013;5:194re2.

27. Palmqvist S, Mattsson N, Hansson O; Alzheimer's Disease Neuroimaging Initiative. Cerebrospinal fluid analysis detects cerebral amyloid- $\beta$ accumulation earlier than positron emission tomography. Brain 2016;139:1226-1236.

28. Buerger K, Ewers M, Pirttila T, et al. CSF phosphorylated tau protein correlates with neocortical neurofibrillary pathology in Alzheimer's disease. Brain 2006;129:3035-3041.

29. Tapiola T, Alafuzoff I, Herukka SK, et al. Cerebrospinal fluid \{beta\}-amyloid 42 and tau proteins as biomarkers of Alzheimer-type pathologic changes in the brain. Arch Neurol 2009;66:382-389.

30. Seppälä TT, Nerg O, Koivisto AM, et al. CSF biomarkers for Alzheimer disease correlate with cortical brain biopsy findings. Neurology 2012;78:1568-1575.

31. Smith R, Wibom M, Olsson T, et al. Posterior accumulation of tau and concordant hypometabolism in an early-onset Alzheimer's disease patient with presenilin-1 mutation. J Alzheimers Dis 2016;51:339-343.

32. Engelborghs S, Sleegers K, Cras P, et al. No association of CSF biomarkers with APOEepsilon4, plaque and tangle burden in definite Alzheimer's disease. Brain 2007; 130:2320-2326.

33. McKhann GM, Knopman DS, Chertkow H, et al. The diagnosis of dementia due to Alzheimer's disease: recommendations from the National Institute on AgingAlzheimer's Association workgroups on diagnostic guidelines for Alzheimer's disease. Alzheimers Dement 2011;7:263-269.

34. Dubois B, Feldman HH, Jacova C, et al. Advancing research diagnostic criteria for Alzheimer's disease: the IWG-2 criteria. Lancet Neurol 2014;13:614-629. 


\section{Comparing "F-AV-1451 with CSF t-tau and p-tau for diagnosis of Alzheimer disease}

Niklas Mattsson, MD, PhD, Ruben Smith, MD, PhD, Olof Strandberg, PhD, Sebastian Palmqvist, MD, PhD, Michael Schöll, PhD, Philip S. Insel, Douglas Hägerström, MD, PhD, Tomas Ohlsson, PhD, Henrik Zetterberg, MD, PhD, Kaj Blennow, MD, PhD, Jonas Jögi, MD, PhD, and Oskar Hansson, MD, PhD Cite as: Neurology ${ }^{\circledR}$ 2018;90:e388-e395. doi:10.1212/WNL.0000000000004887

\author{
Correspondence \\ Dr. Mattsson \\ niklas.mattsson@med.lu.se \\ or Dr. Hansson \\ oskar.hansson@med.lu.se
}

\section{Study question}

How do CSF tau measurements and ${ }^{18} \mathrm{~F}-\mathrm{AV}-1451$ PET imaging of tau pathology compare in utility for diagnosing Alzheimer disease $(\mathrm{AD})$ ?

\section{Summary answer}

CSF tau measurements and ${ }^{18} \mathrm{~F}-\mathrm{AV}-1451$ PET are equally useful for diagnosing early-stage $\mathrm{AD}$, but ${ }^{18} \mathrm{~F}-\mathrm{AV}$-1451 PET is superior for dementia-stage $\mathrm{AD}$.

\section{What is known and what this article adds}

CSF-based and PET-based measurements of tau pathology are both useful for diagnosing $\mathrm{AD}$, but no previous study has directly compared them. This study provides Class III evidence for their relative utilities in different $\mathrm{AD}$ stages.

\section{Participants and setting}

This study recruited 30 healthy controls, 14 patients with prodromal $\mathrm{AD}$, and 39 patients with dementia-stage $\mathrm{AD}$ from the Swedish BioFINDER study. All participants were at least 60 years old.

\section{Design, size, and duration}

CSF samples were collected via lumbar puncture and analyzed for total tau ( $\mathrm{t}$-tau) and phosphorylated tau ( $\mathrm{p}$-tau) with standard procedures. PET scans were used to measure ${ }^{18} \mathrm{~F}-\mathrm{AV}$-1451 standardized uptake value ratios (SUVRs) into literature-defined regions of interest. MRI measures of hippocampal volume and temporal cortex thickness also were obtained. All $\mathrm{AD}$ diagnoses were confirmed by dementia specialists blinded to CSF and PET results. Cutoffs were defined by the Youden index.

\section{Main results and the role of chance}

For differentiating patients with prodromal $\mathrm{AD}$ from controls, the diagnostic accuracies (area under the receiver operating characteristic curve [AUC]) did not differ between ${ }^{18}$ F-AV-1451 and CSF tau biomarkers. The diagnostic sensitivities and specificities were $86 \%$ and $70 \%$, respectively, for CSF t-tau $>504 \mathrm{ng} / \mathrm{L} ; 79 \%$ and 93\%, respectively, for CSF p-tau $>73 \mathrm{ng} / \mathrm{L}$; and $57 \%$ and $100 \%$, respectively, for an ${ }^{18} \mathrm{~F}-\mathrm{AV}-1451$ tau stage I-V SUVR $>1.43$. For distinguishing patients with dementia-stage $\mathrm{AD}$ from controls, the AUCs were greater for ${ }^{18} \mathrm{~F}-\mathrm{AV}-1451$ than for CSF tau biomarkers.
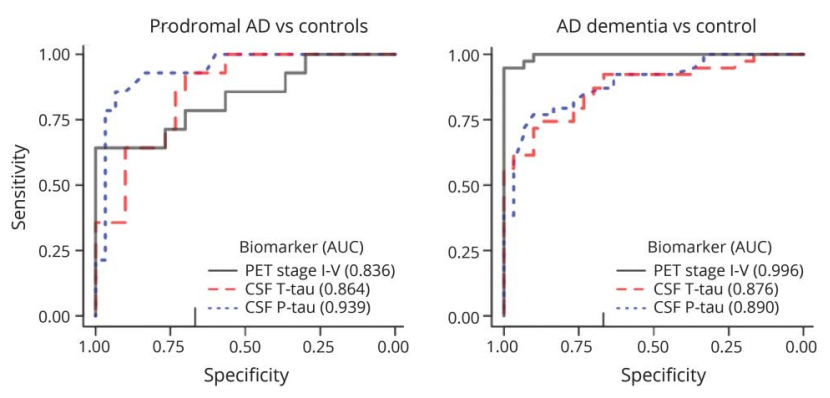

The diagnostic sensitivities and specificities were $69 \%$ and $90 \%$, respectively, for CSF t-tau > $624 \mathrm{ng} / \mathrm{L} ; 72 \%$ and $93 \%$, respectively, for CSF p-tau > $72 \mathrm{ng} / \mathrm{L}$; and $92 \%$ and $100 \%$, respectively, for an ${ }^{18}$ F-AV-1451 tau stage I-V SUVR > 1.37. MRI measures were less robust in both prodromal disease and dementia.

\section{Bias, confounding, and other reasons for caution}

The CSF and PET assessments are both potentially subject to measurement errors. The cerebellar gray matter was used as a PET reference region, but it may not be the optimal reference region. Pathology in these patients was not autopsy-confirmed, and confounding copathology could not be ruled out.

\section{Generalizability to other populations}

This study's patients with $\mathrm{AD}$ all had biomarker evidence of amyloid pathology; therefore, the results may not be generalizable to patients without such evidence.

\section{Study funding/potential competing interests}

This study was funded by the EU and Swedish governments and by various medical research foundations. Some authors report receiving advisory board appointments, consultancy work, and funding from various pharmaceutical and biotechnology companies. Drs. Zetterberg and Blennow cofounded Brain Biomarker Solutions. Go to Neurology.org/N for full disclosures. 


\section{Neurology}

Comparing ${ }^{18} \mathrm{~F}$-AV-1451 with CSF t-tau and p-tau for diagnosis of Alzheimer disease Niklas Mattsson, Ruben Smith, Olof Strandberg, et al.

Neurology 2018;90;e388-e395 Published Online before print January 10, 2018

DOI 10.1212/WNL.0000000000004887

This information is current as of January 10, 2018

\section{Updated Information \&} Services

References

Citations

Subspecialty Collections

Permissions \& Licensing

Reprints including high resolution figures, can be found at: http://n.neurology.org/content/90/5/e388.full

This article cites 33 articles, 8 of which you can access for free at: http://n.neurology.org/content/90/5/e388.full\#ref-list-1

This article has been cited by 4 HighWire-hosted articles: http://n.neurology.org/content/90/5/e388.full\#\#otherarticles

This article, along with others on similar topics, appears in the following collection(s):

Alzheimer's disease

http://n.neurology.org/cgi/collection/alzheimers_disease

Cerebrospinal Fluid

http://n.neurology.org/cgi/collection/cerebrospinal_fluid

Diagnostic test assessment

http://n.neurology.org/cgi/collection/diagnostic_test_assessment_ MCI (mild cognitive impairment)

http://n.neurology.org/cgi/collection/mci_mild_cognitive_impairment PET

http://n.neurology.org/cgi/collection/pet

Information about reproducing this article in parts (figures,tables) or in its entirety can be found online at:

http://www.neurology.org/about/about_the_journal\#permissions

Information about ordering reprints can be found online:

http://n.neurology.org/subscribers/advertise

Neurology ${ }^{\circledR}$ is the official journal of the American Academy of Neurology. Published continuously since 1951, it is now a weekly with 48 issues per year. Copyright @ 2018 The Author(s). Published by Wolters Kluwer Health, Inc. on behalf of the American Academy of Neurology. All rights reserved. Print ISSN: 0028-3878. Online ISSN: 1526-632X.

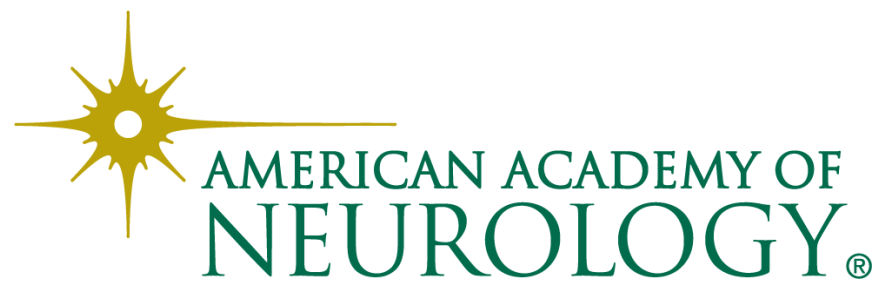

